\title{
DESIGNING AND ANALYSING A TECHNIQUE TO SWITCH ON/OFF BASE STATIONS FOR GREEN CELLULAR NETWORKS
}

\author{
Weston Mwashita ${ }^{1}$, Marcel Ohanga Odhiambo ${ }^{2}$ \\ ${ }^{1}$ MTech student, College of Science, Engineering and Technology, University of South Africa, Windhoek, Namibia \\ ${ }^{2}$ Head of Department, Department of Process Control \& Computer Systems, Faculty of Engineering and Technology, \\ Vaal University of Technology, Andries, Portgieter, South Africa
}

\begin{abstract}
There has been an astronomical increase in data traffic demand as people want to get connected whilst they are on the move and as new devices like smart phones and tablets become affordable gadgets. Mobile network providers have responded to this growth by expanding their networks and naturally this has seen a rapid increase in Base Stations (BSs) all over the world. This trend has led to the telecommunications sector contributing a sizable percentage of $\mathrm{CO}_{2}$ emissions to the atmosphere. Designing Base stations that consume energy corresponding to network traffic will go a long way in reducing $\mathrm{CO}_{2}$ emissions. In this paper, an algorithm that can be used to switch ON/OFF BSs with the traffic variation is designed and analysed.
\end{abstract}

Keywords: Base station sleeping, energy efficiency, Base station-user association, Quality of Service (QoS).

\section{INTRODUCTION}

The demand for data and connectivity has seen an exponential growth in demand for wireless services and this has forced mobile network operators to expand their networks drastically to cater for this demand. In a cellular network, most of the energy is consumed by a Base Station (BS). Authors in [1] put this at between $60 \%$ and $80 \%$. It is in light of this that techniques aimed at making the BS more energy efficient tend to yield good results. Techniques that cause the BS to intelligently switch ON/OFF to follow the network traffic variation are considered very promising in reducing energy consumed by a cellular network and in the process reduce $\mathrm{CO}_{2}$ emissions making the world a better place to live in. Situations like the one shown in figure 1 which is caused by climate change have to be prevented at all costs.

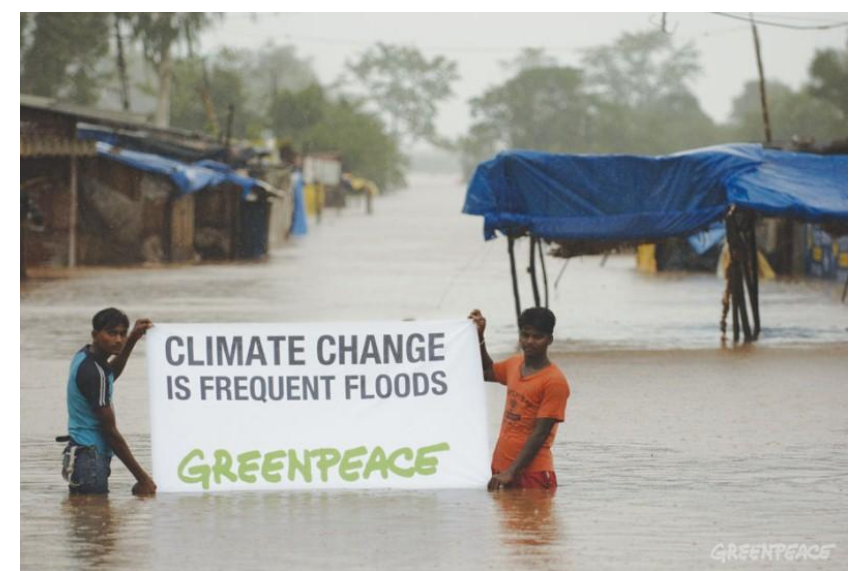

Fig 1: Flood ravaged victims in Navsari, Gujarat, holding a Greenpeace banner acknowledging the fact that severe flooding is an effect of climate change [2]
The ICT industry, with the Telecommunications sector included, contributes about $3 \%$ of Greenhouse Gas (GHG) emissions [3]. These GHG emissions are the ones that are causing the global average temperature to increase annually by between $2 \%$ to $6 \%$ [4]. The Telecommunications sector should be seen to be doing something towards the reduction in the GHG emissions.

\section{RELATED WORK}

Several BS sleeping schemes have been suggested by various researchers. Researchers in [5] proposed a scheme that involves a BS being switched $\mathrm{ON}$ and OFF at preset periods based on the daily traffic pattern. According to these researchers, cellular traffic is affected mainly by two things:

1. The almost routine movement by users in the morning to their work places in town and the movement back to their homes after they knock off at work.

2. The typical day-night behavior of network users.

The researchers went on to show that their BS sleeping solution can save at least $25 \%$ which is quite a substantial saving. However, QoS can be compromised at some instances. The sinusoidal traffic profile as suggested by the authors can at times be disrupted. Events like sporting functions, social gatherings, holidays etc, can disrupt the sinusoidal traffic profile as suggested. These disruptions are most of the time irregular. With this method where BSs are switched ON and OFF at fixed times, the QoS will be affected when the situation deviates from the norm. The algorithm does not take care of the instantaneous user variation and this might lead users of the network to become unhappy when there is a degradation of QoS.

Authors in [6] proposed a switch ON/OFF algorithm that does not switch OFF a BS according to the traffic load of the $\mathrm{BS}$ in question but according to the average distance of 
its users. The BS estimates the distance of its users and also users of its neighbouring BSs. This distance information is then shared amongst the BSs themselves and the algorithm proposed by the researchers switches OFF the BS with the maximum average distance.

The proposal does not attempt to solve the association problem. If a BS is switched OFF, current users within the cell served by that BS need to be redirected to neighbouring BSs to guarantee an acceptable QoS. The BSs to which the current users must be redirected might not necessarily be the nearest BSs. BSs serving the least traffic might be the ones that offer the acceptable QoS. The authors however produced results indicating a $29 \%$ energy saving on implementation of their algorithm. This is quite a huge energy saving but what was not thoroughly investigated was the issue of QoS. A proper association mechanism is necessary to fully exploit the amount of energy saving from traffic aware BS operations.

In [7], a scheme is proposed which adaptively adjusts the cell size according to the prevailing cellular traffic conditions. Cell zooming actively changes the sizes of cells by adjusting the transmit power of control signals and effectively adjusting cell sizes in the process as shown in figure 2. Antenna height and antenna tilt of BSs adjustments can also be used for cells to zoom in or zoom out.

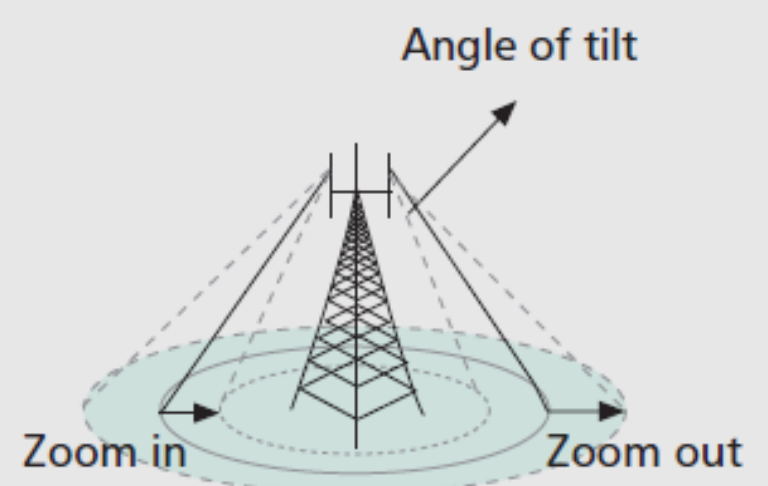

Fig 2: Zooming in and out by reducing transmit power and adjusting antenna tilt [14]

This scheme however has its shortcomings, the major one being that of inter-cell interference and coverage holes. The proposed algorithms; centralised and distributed algorithms also do not adequately address the problem of traffic imbalance.

Authors in [8] proposed a distributed switching OFF algorithm that is designed in such a way that signal strength and system load parameters are shared amongst the BSs and UEs. Using this shared information, each BS then decides whether to switch OFF or to remain ON. There is no centralised controller. The BS that would have decided to switch OFF first has to broadcast a request to switch OFF (RTSO) and will only get to switch OFF upon receiving a clear to switch off (CTSO) signal from neighbouring BSs.
This is done to prevent overlapping neighbouring BSs from simultaneously switching OFF leading to their neighbours having to deal with a traffic overload condition. Since the switching ON and OFF is not centrally controlled, there is a strong possibility of having coverage holes in the cellular network. Also in the event that the RTSO and CTSO are exchanged simultaneously, the proposed algorithm might operate ineffectively leading to a compromised QoS.

Quite a number of BS sleeping algorithms have been suggested. In [9] authors proposed a method of progressively reducing the BS transmit power. They called this technique cell wilting or cell progressive switch OFF. This technique prevents or minimizes any interruption of ongoing calls/sessions. During the process of coverage shrinkage, if any UE experiences unacceptable QoS degradation without the possibility to migrate to neighbouring $\mathrm{BSs}$, it can alert the $\mathrm{BS}$ that is undergoing wilting process for the BS to stop doing so. This technique also results in huge energy savings. Its main problem arises when a UE is switched ON in an area where a BS is in the sleep mode. The BS is OFF and is not in a position to detect this UE. UE has no means to signal its problem since it's in an area that has no coverage. The algorithm that was advanced by these researchers cannot handle coverage holes. QoS can be affected by abrupt changes in transmitted power levels. A UE that receives a strong signal from a nearby BS cannot detect other BSs' signals and if this BS were to switch OFF too quickly, this would result in the UE losing its connection. The proposed algorithm does not have a solution as to how this sudden disconnection can be handled.

Authors in [10] proposed a solution that includes BS sleep control and use of a combination of the conventional BSs that are connected to the power grid and others that are powered by solar and wind. The renewable powered BSs are forced into sleep mode in the absence of enough harvested energy in the battery. The grid powered BS on the other hand, goes into sleep mode when traffic goes very low. The system needs a fairly complex mechanism to coordinate the two sleep modes such that energy outages do not occur. Energy outages can occur in instances where energy stored by renewable energy BSs cannot support UEs that would have been handed over to grid powered BSs that would have gone into sleep mode. The proposed solution results in moderate energy savings but it requires a huge CAPEX (capital expenses) in the beginning.

Authors in [11] proposed a framework that they called TANGO (Traffic-Aware Network planning and Green Operation) with the aim of increasing energy efficiency with guaranteed coverage. The authors however did not consider randomness of cellular traffic in their proposal. The cell zooming that was proposed does not address the issue of coverage holes that is usually associated with cell zooming. For those BSs that would have been made to go into sleep mode, a wake-up mechanism is not part of the framework proposed. 


\section{PROPOSED BS SLEEPING TECHNIQUE}

Switching OFF BSs manually is not a viable and recommended option. A realistic approach would be to have a centralised controller that monitors network information within a specific reasonably sized area. The controller collects network information on traffic load, user requirements and channel information. A traffic load threshold should be set such that if a BS reaches this value, it is switched OFF with the few users being re-associated with neighbouring BSs. The BSs need not completely shut down as suggested by authors in [12]. If they are to completely shut down, there might be coverage holes when a UE is switched ON in an area where a BS would be OFF. To avoid coverage holes, a BS in sleep mode switches ON through control signals coming through a dedicated backhaul link to participate in a handover process of the user to a neighbouring active BS. There should also be a maximum capacity of BS that is set so that when it is reached, excess traffic is diverted to neighbouring BSs through soft handovers. If there is a BS in sleep mode in the near vicinity, it is awoken and the excess traffic is handed over to the accommodating BS or other neighbours handling a reasonable traffic load.

If traffic conditions change suddenly, BSs should just be awoken through backhaul interface connecting BSs to the core network. The remaining active BSs need not increase their power to cover cells where BSs might have gone into sleep mode. This is not necessary as in the proposed solution; the BSs are not completely switched OFF. Since they are partially switched OFF, it means if a UE arrives in the cell, the receiver section of the BS would pick up the signal and in conjunction with the core network, the BS would be awoken to carry the arriving traffic. It wakes up to participate in the process of handing over that UE to an active neighbouring BS. This is one aspect in which the proposed solution differs with majority schemes that have been advanced to solve the problem. With schemes where BSs have to increase their power to cover neighbouring BSs where BSs might be sleeping, [12, 13, 14, 15] coverage holes are always a problem. This is because BSs have a maximum transmission power and if it so happens that there might be UEs outside a BS that is transmitting at maximum power, then those UEs are not covered. Increasing power also reduces the intended energy savings. Network interference also increases as overlapping areas increase as BSs increase their powers in a bid to reduce coverage holes.

The maximum and minimum thresholds for BSs should not be made uniform for the entire network. A heterogeneous network deployment strategy is being proposed. This means that different BSs are deployed intelligently to serve the network efficiently. BSs meant to serve areas like shopping malls should be those that have very high capacities and cannot have the same thresholds as BSs that serve low density residential areas. Historical daily traffic variation analysis should be used to set these thresholds. The aim of the algorithm being proposed is to try and minimise the number of BSs serving the network at any given point in time but still meeting the QoS requirement.
The proposed BS switch ON/OFF algorithm is achieved with the following steps;

Step 1: The traffic load for all BSs within a seven cell cluster is gathered.

Step 2: The BSs are then arranged in ascending order according to their traffic loads.

Step 3: If a BS's traffic load is below a specified minimum threshold, $\alpha_{t h}$, it has to be switched OFF provided there are some neighbouring BSs that can accommodate the few mobiles the BS would be handling. $\alpha_{t h}$, the minimum threshold is not the same for different types of BSs. If there is no neighbour that can accept any additional load, then switch OFF algorithm is halted. If there are accommodating neighbours, then controller needs to broadcast intention to switch OFF BS. This process triggers the BS-user association algorithm. If the traffic load of neigbouring Bs is all above a prescribed minimum threshold, then the algorithm is halted to be restarted after thirty minutes.

Step 4: QoS is checked after a handover process is done. If the switching OFF procedure results in degradation of QoS, then the algorithm is halted.

The flow diagram in figure 3 best summarises the steps.

\subsection{Base Station-User Association Algorithm}

Users in cells in which BSs are to be put to sleep have to be handed over to neighbouring BSs that can best serve them. This is done taking into consideration the traffic load the users might be currently processing and also the traffic load the neighbouring BSs will be processing. Re-association of users is carried out after analysing the performance of network elements. In general, the most commonly used quality performance metrics used to analyse energy consumption in a network according to $\mathrm{E}^{3} \mathrm{~F}$ in [16] are power per unit area and energy per bit.

\subsubsection{Power per Unit Area}

This is the ratio of network average power usage $(\mathrm{P})$ to network coverage area (A), mathematically expressed as:

$$
\mathrm{ECI}_{\mathrm{P} / \mathrm{A}}=\frac{\mathrm{P}}{\mathrm{A}}\left[\mathrm{W} / \mathrm{m}^{2}\right]
$$

where ECI is the Energy Consumption Index

The ECI gives an overview of the total network power. This performance metric can be used to indicate $\mathrm{CO}_{2}$ emissions. However, it is not so sensitive to traffic load as it increases very slowly with total system throughput.

\subsubsection{Specific Energy SE}

This metric is very sensitive to traffic load. It is because of its sensitivity to system throughput that it is chosen to be the metric to be used in this project for the BS-user association algorithm. It is the amount of energy the network consumes to the total number of bits correctly delivered in the network within an observation period $\mathrm{T}$. 
Mathematically:

$$
\mathrm{SE}=\frac{\mathrm{E}}{\mathrm{B}}=\frac{\mathrm{P}}{\mathrm{R}}
$$

Where: $\mathrm{B}$ is the total number of bits

$\mathrm{P}$ is average network power

$\mathrm{R}$ is the data rate

$\mathrm{SE}$ is an indicator of network delivery efficiency. It provides the energy that is required to transport one bit of data in a network. Authors in [17] call it the Energy Consumption ratio (ECR). It can be seen from the formula that as the traffic load in a given cell decreases, the specific energy increases tremendously.

At maximum traffic load, the power that is consumed [18] by a BS is given by

$$
P_{\text {in }}=N_{T R X} \frac{\frac{P_{\text {out }}}{\eta_{P A}\left(1-\sigma_{f a z}\right)}+P_{R F}+P_{B B}}{\left(1-\sigma_{D C}\right)\left(1-\sigma_{M S}\right)\left(1-\sigma_{c o o l}\right)}
$$

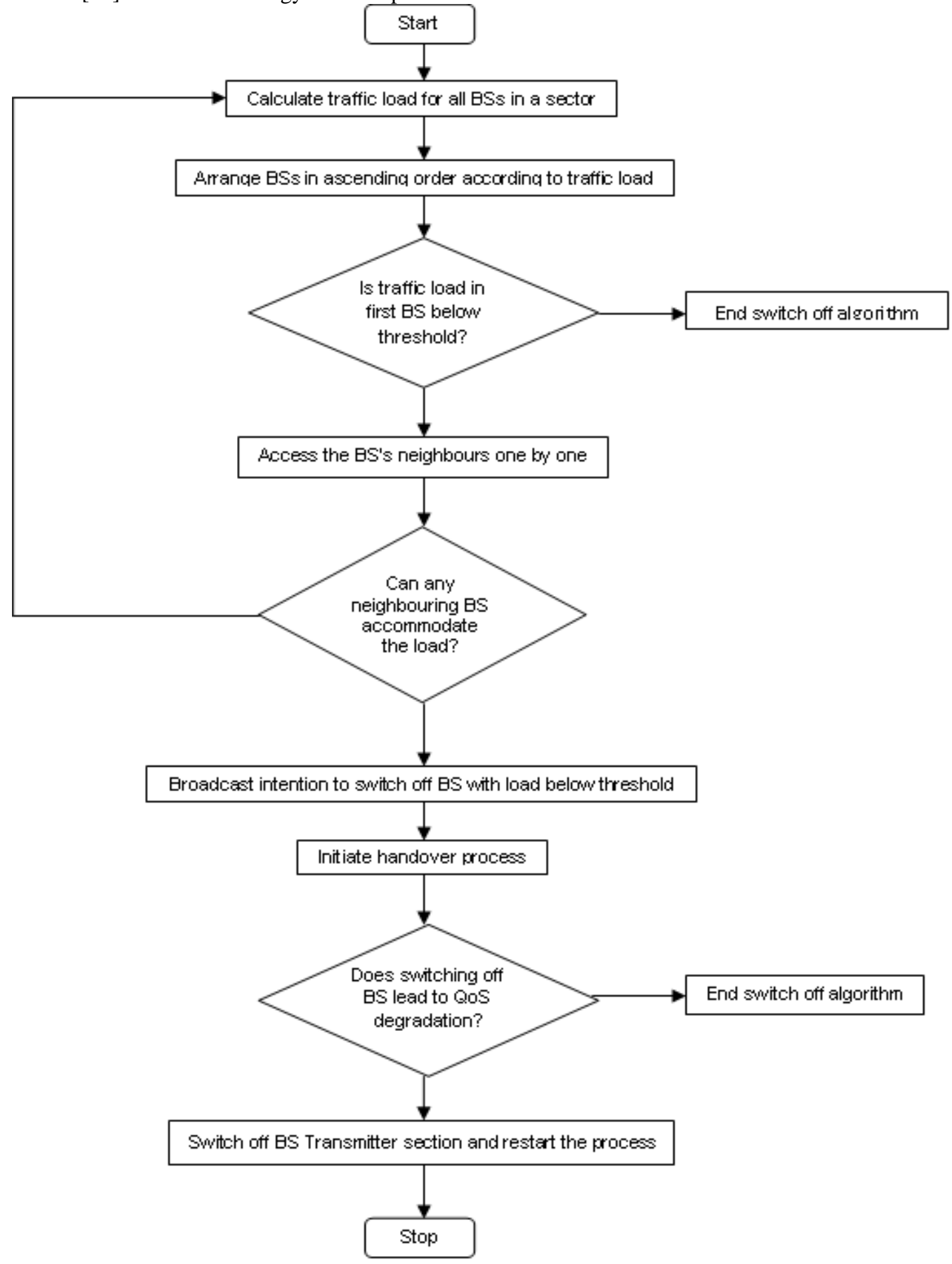

Fig 3: The flow diagram of the proposed BS sleeping algorithm 
Where; $N_{T R X}$ is the number of transceiver chains,

$\sigma \quad$ Is the loss factor and $\sigma=1-\eta$
where $\eta$ is the efficiency given by
$P_{\text {out }} / P_{\text {in }}$

$\begin{array}{ll}\sigma_{\text {feed }} & \text { Represents feeder losses } \\ \sigma_{\text {Cool }} & \text { Represents losses due to cooling system } \\ \eta_{P A} & \text { Represents efficiency of the power } \\ & \text { amplifier } \\ \sigma_{M S} & \text { Losses in the mains supply } \\ \sigma_{D C} & \text { Losses in the DC section of the power } \\ & \text { supply }\end{array}$

According to [19] the overall data rate for one user is given by;

$$
R_{n}=\sum_{k=1}^{K} r_{n}^{k}
$$

Where $\mathrm{k}$ is the total number of sub channels. Sub channels in LTE can be taken as resource blocks assigned to one user.

For a total of $n$ users;

$$
R_{T}=\sum_{n=1}^{N} R_{n}
$$

The total data rate of all users, $i$, being served by one BS [19] taking into account interference plus noise is given by:

$$
R_{T_{i} i}=\eta_{i} \sum_{n=1}^{N} N_{R B_{i} n} \log _{2}\left(1+\frac{P_{t x}-A L F_{n}}{I N_{n}}\right)
$$

\footnotetext{
Where $\eta_{i}=\eta_{B W} \cdot \eta_{B W_{R B}} \quad\left[\eta_{B W}\right.$ Is system bandwidth and $\eta_{B W_{R B}}$ is resource block Bandwidth efficiency]
}

$\mathrm{IN}_{\mathrm{n}} \quad$ Represents interference plus noise

$\mathrm{N}_{\mathrm{RB}_{2} \mathrm{n}} \quad$ Represents the number of resource blocks allocated to the nth user.

$\mathrm{P}_{\mathrm{tx}} \quad$ Represents the transmitted power

$\mathrm{ALF}_{\mathrm{n}}$ Represents the aggregate signal attenuation factor due to path loss of the nth user
The aggregate signal attenuation factor can be obtained by choosing the appropriate path loss model.

Since;

$$
\mathrm{SE}=\frac{\mathrm{E}}{\mathrm{B}}=\frac{\mathrm{P}}{\mathrm{R}}
$$

Then full SE for one base station serving $n$ users is computed as, combining equations (1) and (4):

$$
\begin{array}{r}
S E=\left(N_{T R X} \frac{\frac{P_{\text {out }}}{\eta_{P A}\left(1-\sigma_{\text {feed }}\right)}+P_{R F}+P_{B B}}{\left(1-\sigma_{D C}\right)\left(1-\sigma_{M S}\right)\left(1-\sigma_{c o o l}\right)}\right) \\
\left(\eta_{i} \sum_{n=1}^{N} N_{R B_{2} n} \log _{2}\left(1+\frac{P_{t x}-A L F_{n}}{N N_{n}}\right)\right)
\end{array}
$$

It should be noted that, even at maximum traffic load, SE cannot assume a maximum value at this point since the number of bits delivered is also maximum at this point. SE roughly indicates how busy a BS is. The SE of a BS whose traffic load is not so high will not also be very high. A high SE points to a low traffic load. A low SE on the other hand represents a high traffic load. When users have to be reassociated with neighbouring BSs, BSs with high SE are the ones to be targeted. This way, not only are users reassociated with neighbouring BSs in an energy efficient manner, but the method ensures good load balancing. Overloading one BS reduces QoS experienced by users being served by that particular BS. Users that need to be transferred to neighbouring BSs are themselves arranged in descending order according to the traffic load they are handling. The one with the heaviest load is the one that is handed over to a BS with the highest SE. This is done so that users with heavy loads are handed over to neighbouring BSs with the least traffic load. This ensures that QoS requirements are easily met.

The BS-user association is undertaken step by step, with the user with the heaviest load being associated with BS with the least traffic load using the SE performance metric, followed by the user with the second heaviest traffic load being paired up with a BS with the second lowest traffic load. The process continues until all users within an-aboutto-sleep BS are all re-associated with neighbouring BSs. This is a fail-safe mechanism to ensure that $\mathrm{QoS}$ is not compromised.

The BS switching ON/OFF algorithm also has a mechanism to ensure a high QoS is maintained during the time lowly loaded BSs are being switched OFF. The process of reassociation is triggered when the central controller broadcasts intention to switch OFF a BS. 
In short, the steps taken are as follows:

Step 1: BSs that are neighbours to the BS that has to be switched OFF calculate their SE and send this information to the controlling element.

Step 2: The controlling element arranges the SEs from the neighbouring BSs in descending order

Step 3: UEs that need to be handed over to neighbouring BSs are arranged in descending order according to the traffic they are handling, which means the UE with heaviest load will be on top of the list. The BS on the top of the list is paired with the UE on the top. If a BS can serve a UE according to the pairings, then handover process is accepted otherwise the $\mathrm{BS}$ is prevented from switching OFF. It might happen that a heavy user, if handed over to a BS, causes the recipient BS to be driven into an overload condition; this has to be prevented from occurring as it compromises the QoS. The BS-user association is summarised by the flow diagram given in figure 4 .

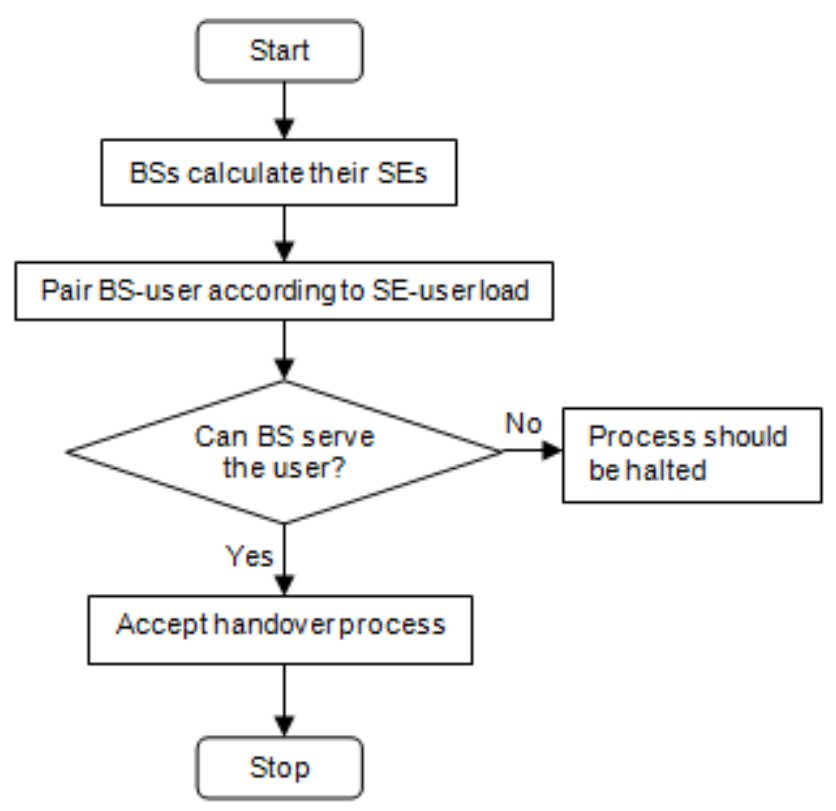

Fig 4: The BS-user association flow diagram

In LTE-Advanced, according to 3GPP specifications in the latest release 13 of 2015, heterogeneous networks make use of Relay nodes (RNs) [20] to provide enhanced coverage to boost capacity at all edges including hotspots. RNs are connected wirelessly via the radio interface to the donor eNB (DeNB) as shown in figure 5.

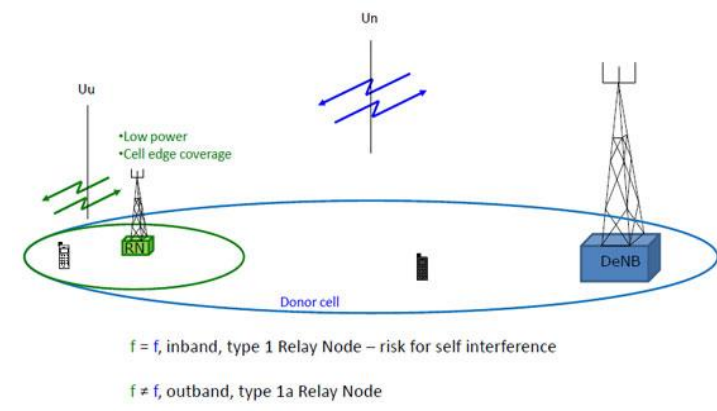

Fig 5: A donor cell with a Relay Node inside [20]
Future networks making use of this concept should also be catered for by the proposed algorithm. At times around the hotspots, the number of users might go below the threshold such that the few UEs remaining have to be handed to over to the DeNB or vice versa. Soft handovers take place if the $\mathrm{Un}$ and $\mathrm{Uu}$ interfaces (interfaces shown in figure 5) are using the same frequency, If the frequencies are different, then hard handovers take place.

\section{CONCLUSIONS AND FUTURE WORK}

A technique that can be used to switch ON/OFF BSs has been designed and analysed. For our future work the proposed BS sleeping algorithm has to be tested on homogeneous as well as heterogeneous networks. It then has to be fine tuned so that it can work well with heterogeneous networks as heterogeneous networks are networks of the future.

\section{REFERENCES}

[1] R. Murthy, \& C. Kavitha, "A survey of Green base stations in cellular networks, "IRACST International Journal of Computer Networks and Wireless Communications, (IJCNWC), vol.2, no.2, 2012, pp. 232-236.

[2] S. Rahut \& S. Bhattacharjee, "Climate change is floods: Greenpeace warns as rains ravage Mumbai," July $62007 . \quad$ [Online] Available: http://www.greenpeace.org/india/en/news/climatechange-is-frequent-flo/

[3] M.H Asharif, R.Nordin \& M. Ishmail, "A survey of green radio communication Networks: Techniques and recent advances," Journal of computer networks and communications, vol. 2013, Article 45389, Hindawi Publishing Corporation, 2013.

[4] J. Houghton, "Global warming, climate change and sustainability: Challenge to scientists, policy makers and Christians," The John Ray Initiative, briefing paper 14, 2011.

[5] M.A. Marsan, L. Chiaraviglo, D. Ciulo \& M. Meo, "Optimal energy savings in cellular access networks, "IEEE Green communication, Dresden, 2009.

[6] A. Bousia, A. Antonopoulos, L.Alonso \& C. Verikoukis, "“Green” Distance-aware base station sleeping algorithm in LTE-advanced." [Online] Available: http://cveri.es/images/d/da/Alex_ICC_12.pdf

[7] Z. Niu, Y. Wu, J. Gong \& Z. Yang, "Cell zooming for cost-efficient Green Cellular Networks," [Online].Available:

http://www.network.ee.tsingua.edu.cn/papers/NiuC ommag10.pdf

[8] K. Son \& B. Krishnamachari, "Dynamic base station switching-on/off Strategies for green cellular networks," IEEE transactions on wireless communications, vol. xx no. xx, 2013.

[9] A. Conte, A.Feki, L.Chiaraviglo, D. Ciullo, M. Meo \& M.A. Marsan," Cell wilting And blossoming for energy efficiency,"2013, [Online].Available: 
www.telematica.polito.it/oldsite/chiaraviglo/papers /wilting_Blossoming.pdf

[10] S. Zhou, J. Gong \& Z. Niu, "Sleep control for base stations powered by heterogeneous energy sources," [Online]. Available: https://researchgate.net/publication/261244515_Sleep _control_for_base_stations_powered_by_heterogene ous_energy_sources

[11] Z. Niu, "TANGO: traffic-aware network planning and green operation," IEEE Wireless Communications, vol., 18, no. 5, 2011,pp.25-29.

[12] K. Son \& B. Krishnamachari, "Dynamic base station switching-on/off Strategies for green cellular networks," IEEE transactions on wireless communications, vol. xx no. xx, pp. 1-11, 2013.

[13] M.A Marsan, L. Chiaraviglo, D. Ciulo \& M. Meo, "Optimal energy savings in cellular access networks," Communications Workshops,2009, ICC Workshops 2009. IEEE International Conference on, pp. 1-5, Jun. 2009.

[14] A. Conte, A. Feki, L. Chiaraviglo, D. Ciullo, M. Meo \& M.A. Marsan, "Cell wilting and blossoming for energy efficiency," Wireless Communications, IEEE, vol. 18, no. 5, pp. 50-57. Oct. 2011.

[15] Z. Niu, Y. Wu, J. Gong \& Z. Yang. "Cell zooming: for cost-efficient green cellular networks," Communications Magazine, IEEE, vol. 48, no. 11, pp. 74-79, Nov. 2010.

[16] G. Auer, V. Giannini, C. Desset, I. Godor, P. Skillermark, M. Olsson, M. A. Imran \& D. Sabella, "How much energy is needed to run a wireless network?," Wireless Communications, IEEE, vol. 18, pp. 40-49, Oct. 2011.

[17] Y. Fan, X. Wang \& C.H.J Peter. (2010). Green Cellular....Towards Sustainable Networks. [Online]. Available:

www.mobile.ecei.tohoku.ac.jp/COE/seminar_2010_0 6_2/Green.pdf

[18] G. Auer, V. Giannini, C. Desset, I. Godor, P. Skillermark, M. Olsson, M. A. Imran \& D. Sabella, O. Blume, W. Wajda, Y. Jading \& E. Katranaras ,"Energy efficiency analysis of the reference systems, areas of improvements and target breakdown," [Online].

Available: http://cordis.europa.eu/docs/projects/cnet/3/247733/0 80/deliverables/001-EARTHWP2D23v

[19] P. Dini, M. Miozzo, N. Bui \& N. Baldo, "A model to analyze the energy savings of base station sleeping in LTE HetNets," 2013 IEEE International Conference on Green computing and communications and IEEE Internet of Things and IEEE Cyber, Physical and Social Computing, pp. 1375-1380, 20-23 Aug. 2013.

[20] J. Wannstrom, "The mobile broadband standard," [Online] Available: http://www.3gpp.org/technologies/keywordsacronyms/97-lte-advanced

\section{BIOGRAPHIES}

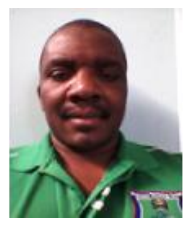

Weston Mwashita is a post graduate student at University of South Africa who is currently seized with MTech: Engineering: Electrical specializing in telecommunications. He is also lecturing Avionics, Electronics, Telecoms subjects at NAF TTC, Ministry of Defence in Windhoek, Namibia. PO Box 5172Windhoek, Namibia. 47955864@mylife.unisa.ac.za

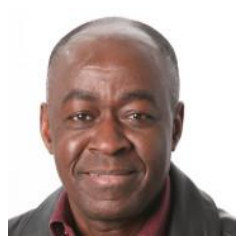

Prof. Marcel Ohanga Odhiambo is the Head of the Process Control and Computer Systems at Vaal University of Technology in South Africa. He is a renowned researcher who has contributed immensely to the fields of Engineering and Technology. Private Bag X021 Vanderbijlpark,1911, Andries, Portgieter, South Africa marcelo@vut.ac.za, 